\title{
Nouvelles recommandations du CCNI en matière de prophylaxie post-exposition contre la rougeole
}

\author{
MC Tunis ${ }^{1}$, MI Salvadori ${ }^{2}, V_{\text {Dubey }}{ }^{3}$, O Baclic ${ }^{1}$ au nom du Comité consultatif national de \\ I'immunisation (CCNI)*
}

\section{Résumé}

Contexte : Les produits à base d'immunoglobuline (lg) humaine sont actuellement recommandés pour la prophylaxie post-exposition (PPE) contre la rougeole dans certains groupes susceptibles. Cependant, le succès des programmes de vaccination contre la rougeole en Amérique du Nord a entraîné une faible circulation du virus de la rougeole et la plupart des donneurs de sang n'ont maintenant qu'une immunité vaccinale. Concurremment, les concentrations d'anticorps anti-rougeole des produits à base d'Ig humaine ont démontré une tendance de diminution progressive. De plus, les doses et les voies d'administration auparavant recommandées n'offrent peut-être plus une protection optimale.

Objectifs : Effectuer une recension des écrits et mettre à jour les dernières recommandations sur la prophylaxie post-exposition contre la rougeole, y compris la posologie et les voies d'administration pour l'Ig rougeoleuse en PPE, chez les nourrissons susceptibles et les personnes immunodéprimées ou enceintes, afin de prévenir la forme grave de la maladie.

Démarche : Le groupe de travail sur la rougeole, la rubéole, les oreillons et la varicelle (RROV) du Comité consultatif national de l'immunisation (CCNI) a revu les écrits clés, les pratiques internationales et les renseignements sur les produits à base d'lg actuels en ce qui concerne la dose optimale et les voies d'administration des produits à base d'lg à utiliser dans le cadre de la PPE contre la rougeole. Le groupe a ensuite proposé des changements aux recommandations sur la PPE fondés sur des données probantes, qui ont été évalués et approuvés par le CCNI.

Résultats : Le CCNI continue de recommander l'administration du vaccin contre la rougeole, la rubéole et les oreillons (RRO) dans les 72 heures qui suivent l'exposition à la rougeole pour les personnes susceptibles et immunocompétentes qui sont âgées de six mois et plus ne présentant aucune contre-indication au vaccin. Pour les nourrissons susceptibles de moins de six mois, lorsque le volume d'injection n'est pas une préoccupation importante, le CCNI recommande l'administration d'immunoglobuline par voie intramusculaire (Ig $I M)$ à une concentration de $0,5 \mathrm{~mL} / \mathrm{kg}$, jusqu'à une dose maximale de $15 \mathrm{~mL}$ administrée par plusieurs sites d'injection. Les nourrissons susceptibles de six à 12 mois dont l'infection est décelée après 72 heures et dans les six jours suivant l'exposition à la rougeole devraient recevoir des $\mathrm{lg} I \mathrm{IM}$ (à raison de $0,5 \mathrm{~mL} / \mathrm{kg}$ ) si le volume d'injection n'est pas une préoccupation importante. Également si le volume d'injection n'est pas une préoccupation importante, les personnes susceptibles qui sont enceintes ou immunodéprimées peuvent recevoir des $\mathrm{lg} \mathrm{IM}$ à raison de $0,5 \mathrm{~mL} / \mathrm{kg}$, tout en tenant compte que les personnes de $30 \mathrm{~kg}$ ou plus ne recevront pas les concentrations d'anticorps contre la rougeole qui sont jugées nécessaires pour offrir une protection complète. Alternativement, lorsque le volume d'injection n'est pas une préoccupation importante ou chez les personnes de $30 \mathrm{~kg}$ ou plus, l'immunoglobuline peut être administrée par voie intraveineuse (lg IV) à une dose de $400 \mathrm{mg} / \mathrm{kg}$.

Le CCNI ne recommande pas la PPE par Ig contre la rougeole chez les personnes susceptibles immunocompétentes de plus de 12 mois en raison du faible risque de complications liées à la maladie et de les défis cliniques liés à l'administration pour la prise en charge des cas et des contacts.

Conclusion : Le CCNI a mis à jour les recommandations en matière de PPE contre la rougeole en tenant compte des données probantes actuelles et des pratiques exemplaires pour prévenir la forme grave de la maladie au Canada. Ces recommandations, conformes à celles établies dans d'autres pays, tiennent compte de l'utilisation non approuvée des Ig IV dans certains cas.

Citation proposée : Tunis MC, Salvadori MI, Dubey V, Baclic O au nom du Comité consultatif national de l'immunisation (CCNI). Nouvelles recommandations du CCNI en matière de prophylaxie post-exposition contre la rougeole. Relevé des maladies transmissibles au Canada 2018;44(9):256-61

https://doi.org/10.14745/ccdr.v44i09a07f

Mots-clés : éclosion de rougeole, vaccin contre la rougeole, prophylaxie post-exposition, recommandations du $\mathrm{CCNI}$, immunoglobulines intraveineuses, immunoglobulines intramusculaires, Canada

\section{Affiliations}

${ }^{1}$ Agence de la santé publique du Canada, Centre de l'immunisation et des maladies respiratoires infectieuses, Ottawa (Ontario)

2 Présidente du groupe de travail sur la rougeole, la rubéole, les oreillons et la varicelle, CCNI, London (Ontario)

${ }^{3}$ Vice-présidente du groupe de travail sur la rougeole, la rubéole, les oreillons et la varicelle, CCNI, Toronto (Ontario)

*Correspondance : phac.naciccni.aspc@canada.ca 